NOTE TECHNIQUE

\title{
OBSERVATIONS SUR LE COMPORTEMENT D'ALEVINS DE SAUMON (SALMO SALAR L.) APRÉS LEUR DÉVERSEMENT EN RIVIĖRE
}

\author{
M. LEGAULT*, L.M. LALANCETTE \\ Université du Québec à Chicoutimi. \\ Département des Sciences Fondamentales, \\ 555, Boulevard de l'Université, \\ Chicoutimi (Québec) G7H 2B1, Canada

\begin{abstract}
* Adresse actuelle : Ministère du Loisir, de la Chasse et de la Pêche, Service de l'Aménagement et de l'Exploitation de la Faune, C.P. 1156, Gaspé (Québec) GOC 1RO, Canada
\end{abstract} \\ Reçu le 17 Juin 1986 \\ Accepté le 2 Janvier 1987 \\ Received 17 June, 1986 \\ Accepted 2 January, 1987

\section{RESUME} \\ Des observations effectuées directement sous l'eau ont servi durant deux étés à déterminer les \\ principales causes favorisant la prédation effectuée par l'omble de fontaine (Sa/velinus fontinalis) sur \\ des alevins $(0+)$ de saumons atlantiques (Salmo salar) nouvellement déversés en milieu naturel. Les \\ mauvaises conditions prévalant durant le transport de la pisciculture aux lieux de déversement ainsi \\ qu'une méthode d'introduction inadéquate seraient responsables en grande partie de la prédation \\ effectuée par l'omble de fontaine. Cette prédation pourrait être minimisée en donnant aux alevins une \\ période de récupération et en les dispersant dans des habitats qui répondent à leurs exigences, tout en \\ respectant la capacité d'accueil de ces habitats.
}

\section{OBSERVATIONS ON THE FRIES BEHAVIOR OF ATLANTIC SALMON (SALMO SALAR L.) AFTER THEIR RELEASE IN THE RIVER}

\section{ABSTRACT}

Over two summers we used direct underwater observation to determine the principal causes for the predation of newly planted Atlantic salmon fries (Salmo salar)by brook trout (Salvelinus fontinalis). Bad conditions affecting the transportation of the salmon from the hatchery to the planting sites and an inadequate planting method could be considered responsible for most of the predation by the brook trout. This predation could be prevented by allowing the fries a recuperation period and by scattering them in habitats possessing suitable carrying capacity.

\section{INTRODUCTION}

Le déversement de saumons atlantiques (Salmo salar) juvéniles d'élevage dans les rivières est une pratique d'aménagement courante dans l'est de l'Amérique du Nord. Cependant, leur taux de survie est moins élevé que celui des saumons juvéniles sauvages (ELSON, 1975; LEGAULT. 1985). Selon MC CRIMMON (1954) la mortalité intervient principalement très tôt après l'introduction et elle serait due en grande partie à une prédation par l'omble de fontaine (Salvelinus fontinalis) qui cohabite fréquemment avec le saumon atlantique. Paradoxalement, selon GIBSON (1973), la prédation ne semble pas être importante dans les interactions entre ces deux espèces lorsqu'elles coexistent dans une rivière naturelle, puisqu'elles sont principalement insectivores (BENSON, 1953; KEENLEYSIDE, 1962).

Il existe très peu d'études portant sur le comportement des jeunes saumons et sur leur devenir immédiat, suite à leur introduction dans une rivière naturelle. Les objectifs visés au cours de cette recherche sont donc : (1) observer le comportement d'alevins $(0+)$ de saumons atlantiques au moment de leur déversement et durant les quelques heures qui suivent, afin de déterminer la ou les causes favorisant la prédation effectuée par l'omble de fontaine sur les alevins nouvellement introduits et (2) donner certaines recommandations sur la façon d'effectuer ces introductions dans une rivière dans le but de minimiser la prédation.

Cette étude a été effectuée dans le cadre d'un programme de recherche visant à déterminer les répercussions de l'élevage en pisciculture du saumon atlantique sur son comportement et ses performances dans une rivière naturelle (LEGAULT, 1985). 


\section{MATÉRIEL ET METHODES}

Les observations ont été effectuées en 1982 et 1983 dans la rivière aux Saumons $\left(48^{\circ} 37^{\prime}\right.$ N. : $72^{\circ} 37^{\circ}$ O.), située à l'ouest du lac Saint-Jean, Québec (fig. 1). La rivière aux Saumons est un important tributaire de la rivière Ashuapmushuan. Elle draine sur une distance de $63 \mathrm{~km}$ un bassin versant de $592 \mathrm{~km}^{2}$. La chute $25(8 \mathrm{~m})$ et la chute $50(17 \mathrm{~m})$ localisées respectivement à $21,8 \mathrm{~km}$ et $23,8 \mathrm{~km}$ de l'embouchure bloquent la progression des saumons atlantiques adultes vers l'amont. Les observations ont été effectuées dans la section amont de la rivière (fig. 1) où celle-ci a une largeur moyenne de $24 \mathrm{~m}$ et possède $55 \%$ de zones d'eaux rapides $\left(>50 \mathrm{~cm} . \mathrm{s}^{-1}\right)$ et peu profondes $(<1 \mathrm{~m})$ et $45 \%$ de zones d'eaux lentes ( $\left.<50 \mathrm{~cm} . \mathrm{s}^{-1}\right)$ et profondes $(>1 \mathrm{~m})$.

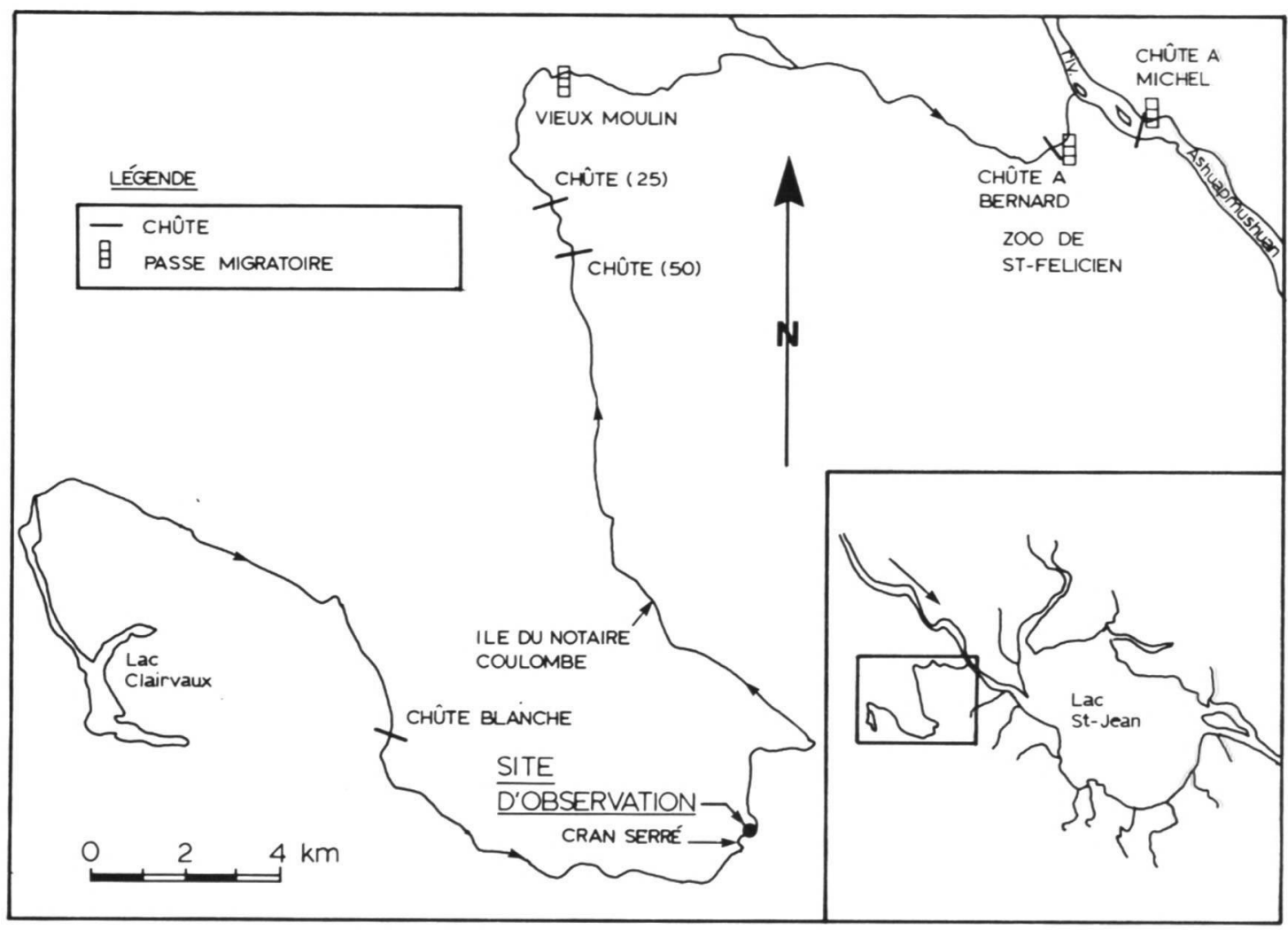

Figure 1 : Localisation de la rivière aux Saumons et site d'observation.

Figure 1 : Location of the Salmon River and sites of observation.

Le site d'observation (fig 2) a été choisi afin d'obtenir les conditions environnementales représentatives des différents micro-habitats rencontrés dans cette rivière. Ce site est composé d'un rapide d'environ $400 \mathrm{~m}$, divisé en deux dans sa partie aval par un amoncellement rocheux. Le rapide est délimité en amont par une section d'eau lente et en aval par un bassin de $7.000 \mathrm{~m}^{2} \mathrm{~d}^{\prime}$ une profondeur moyenne de $2,5 \mathrm{~m}$. A l'extrémité aval du bassin on retrouve un petit rapide de $50 \mathrm{~m}$ de longueur suivi d'une section d'eau calme.

Cette section de la rivière possède une population d'ombles de fontaine dont la densité moyenne était de 0,6 poissons. $100 \mathrm{~m}^{-2}$ dans le rapide de la zone d'observation et de 2,4 poissons. $100 \mathrm{~m}^{-2}$ dans le bassin (LEGAULT,1985). Bien qu'il n'y ait pas une population naturelle de saumons dans cette partie de la rivière, des introductions d'alevins de saumons atlantiques d'eau douce étaient effectuées depuis 1980. C'est ainsi qu'il y avait une densité moyenne dans le rapide et le bassin de la zone d'observation de 7,7 et 5,7 tacons. $100 \mathrm{~m}^{-2}$ respectivement. Finalement, 7 espèces de cyprinidés étaient rencontrées dans ce secteur de la rivière.

Au début du mois de juillet de chacune des deux années, 2.000 alevins de saumons atlantiques d'eau douce d'élevage ont été introduits dans le site d'observation. Le transport des poissons s'est effectué par camion des piscicultures gouvernementales de Tadoussac en 1982 et de Baldwin en 1983. La durée totale du transport des poissons a été de 5 à $8 \mathrm{~h}$ respectivement. Les poissons ont été 


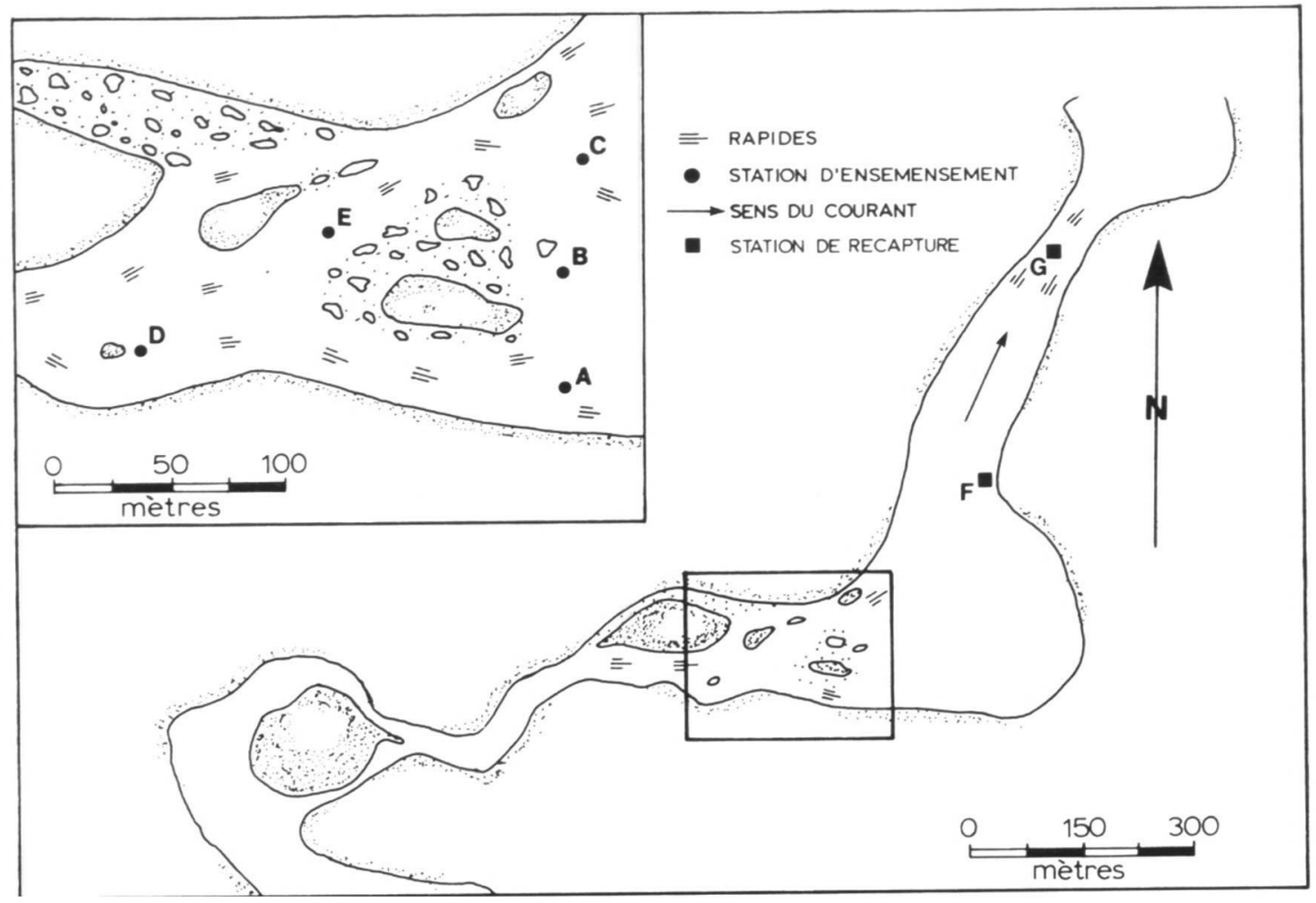

Figure 2 : Zone d'observation et localisation des stations de déversement (A, B, C, D et E) et des lieux de recaptures d'alevins (F et $G$ ).

Figure 2 : Area of observation and location of stocking fries (A, B, C, D and E) and sites of fries recapture (F and $\mathbf{G}$ ).

transportés par groupe de 500 individus dans des sacs de polythène d'une longueur de $122 \mathrm{~cm}$ et de $23 \mathrm{~cm}$ de circonférence. Les sacs contenaient 6 ld'eau, étaient gonflés d'oxygène pur et étaient gardés froids avec de la glace. Les alevins de saumons introduits en 1982 avaient une longueur moyenne à la fourche de $3,4 \mathrm{~cm}$ et provenaient de géniteurs, des ouananiches (saumons d'eau douce), capturés en 1981 dans la rivière Métabetchouane, Québec. En 1983. les alevins provenaient de la souche domestique de saumons d'eau douce de la pisciculture de Baldwin et avaient une longueur moyenne à la fourche de $3,5 \mathrm{~cm}$. L'origine et les caractéristiques physiques des poissons utilisés sont données au tableau 1. Les alevins ont été introduits par groupes de 500 individus aux stations A, B, C et D en 1982

\begin{tabular}{|c|c|c|c|c|}
\hline $\begin{array}{l}\text { Date du } \\
\text { déversement }\end{array}$ & Origine & $\operatorname{LF}(\sigma)$ & $P(\tau)$ & $k(0)$ \\
\hline ? juillet 1982 & $\begin{array}{l}\text { Parents } \\
\text { sauvages }\end{array}$ & $3,4(0,2)$ & $0,4(0,1)$ & $1,02(0,08)$ \\
\hline 29 juin 1983 & Domestiques $^{b}$ & $3,5(0,4)$ & $0,6(0,2)$ & $1,23(0,13)$ \\
\hline
\end{tabular}

a Parents sauvages de la rivière Métabetchouane, Quēbec.

b Souche Baldwin B83-76, Québec.

c Spècimens formolés à $5 \%$.

Tableau 1 : Origines et caractéristiques physiques des alevins d'élevage déversés ${ }^{c}$ en 1982 et 1983. LF : longueur moyenne a la fourche $(\mathrm{cm}), P$ : poids moyen $(\mathrm{g}), \mathrm{K}$ : coefficient moyen de condition et $\sigma$ : écart type.

Table 1 : Origin and physical characteristics of fries grown in pisciculture and released in the river in 1982 and 1983. 
et aux stations A, C, D et E en 1983 (fig. 2). Les stations A et C étaient situées au pied du rapide et à proximité du bassin, la station $B$ étant localisée entre les deux bras du rapide où le courant est nul. Les stations $D$ et $E$ étaient situées au centre du rapide. Une description des stations d'étude est donnée au tableau 2.

\begin{tabular}{|c|c|c|c|c|}
\hline Station & Annêe & $\begin{array}{c}\text { Profondeur } \\
(\mathrm{cm})\end{array}$ & $\begin{array}{c}\text { Vitesse du courant }{ }^{a} \\
\left(\mathrm{~cm} \mathrm{~s}^{-1}\right)\end{array}$ & Type de fond ${ }^{b}$ \\
\hline A & $\begin{array}{l}1982 \\
1983\end{array}$ & $\begin{array}{l}35 \\
35\end{array}$ & $\begin{array}{l}51-41 \\
55-52\end{array}$ & $\begin{array}{l}\text { galets, blocs et } \\
\text { sable }\end{array}$ \\
\hline B & 1982 & 40 & $0-0$ & $\begin{array}{l}\text { sable, galets et } \\
\text { blocs }\end{array}$ \\
\hline $\mathrm{C}$ & $\begin{array}{l}1982 \\
1983\end{array}$ & $\begin{array}{l}40 \\
25\end{array}$ & $\begin{array}{l}43-29 \\
61-52\end{array}$ & $\begin{array}{l}\text { blocs, galets et } \\
\text { sable }\end{array}$ \\
\hline$D$ & $\begin{array}{l}1982 \\
1983\end{array}$ & $\begin{array}{r}65 \\
-\end{array}$ & $\begin{array}{l}45-38 \\
43-18\end{array}$ & $\begin{array}{l}\text { blocs, galets, } \\
\text { cailloux et sable }\end{array}$ \\
\hline$E$ & 1983 & 25 & $42-25$ & $\begin{array}{l}\text { blocs, galets et } \\
\text { cailloux }\end{array}$ \\
\hline
\end{tabular}

a Vitesse du courant mesurée à la surface et au fond du plan d'eau à l'aide d'un courantomètre Gurley no 625.

b Par ordre d'importance relative des différents substrats, selon la classification de Cummins (HYNES, 1972).

Tableau 2 : Description des stations d’étude utilisées lors des observations en apnée en 1982 et 1983.

Table 2 : Description of the stations of released fries used for observations with a snorkel in 1982 and 1983.

Lors des introductions, deux personnes équipées d'un ensemble de plongée en apnée ont observé le comportement des alevins (fig. 3). En 1982, les observations ont été faites le jour de l'introduction et les deux journées suivantes tandis qu'en 1983, les observations ne se sont poursuivies qu'au lendemain de l'introduction. Une séquence d'observation quotidienne était effectuée à chaque station. Les observations effectuées la première année nous ont permis d'évaluer le temps nécessaire pour observer le comportement des alevins lors de leur implantation. Il s'est avéré suffisant d'observer les alevins durant une période de 25 à 30 minutes lors de leur implantation et le lendemain afin d'obtenir une image représentative de leur comportement. Les séquences d'observation par station sont données au tableau 3. L'observateur se déplaçait très lentement aux environs de chaque lieu de déversement, s'immobilisant régulièrement afin d'observer les alevins. Il était ainsi possible d'approcher à moins de $30 \mathrm{~cm}$ d'un poisson avant que celui-ci semble être importuné. La température de l'eau au moment de l'introduction était de $14{ }^{\circ} \mathrm{C}$ en 1982 et de $18{ }^{\circ} \mathrm{C}$ en 1983. Le temps lors des observations de 1982 était nuageux et il y avait des averses, tandis que lors des observations de 1983 le temps était ensoleillé et le ciel était clair. La visibilité pour les deux années d'observation était d'environ $2 \mathrm{~m}$. La durée des observations quotidiennes variait de 80 à 175 minutes et était répartie sur l'ensemble des stations étudiées. 


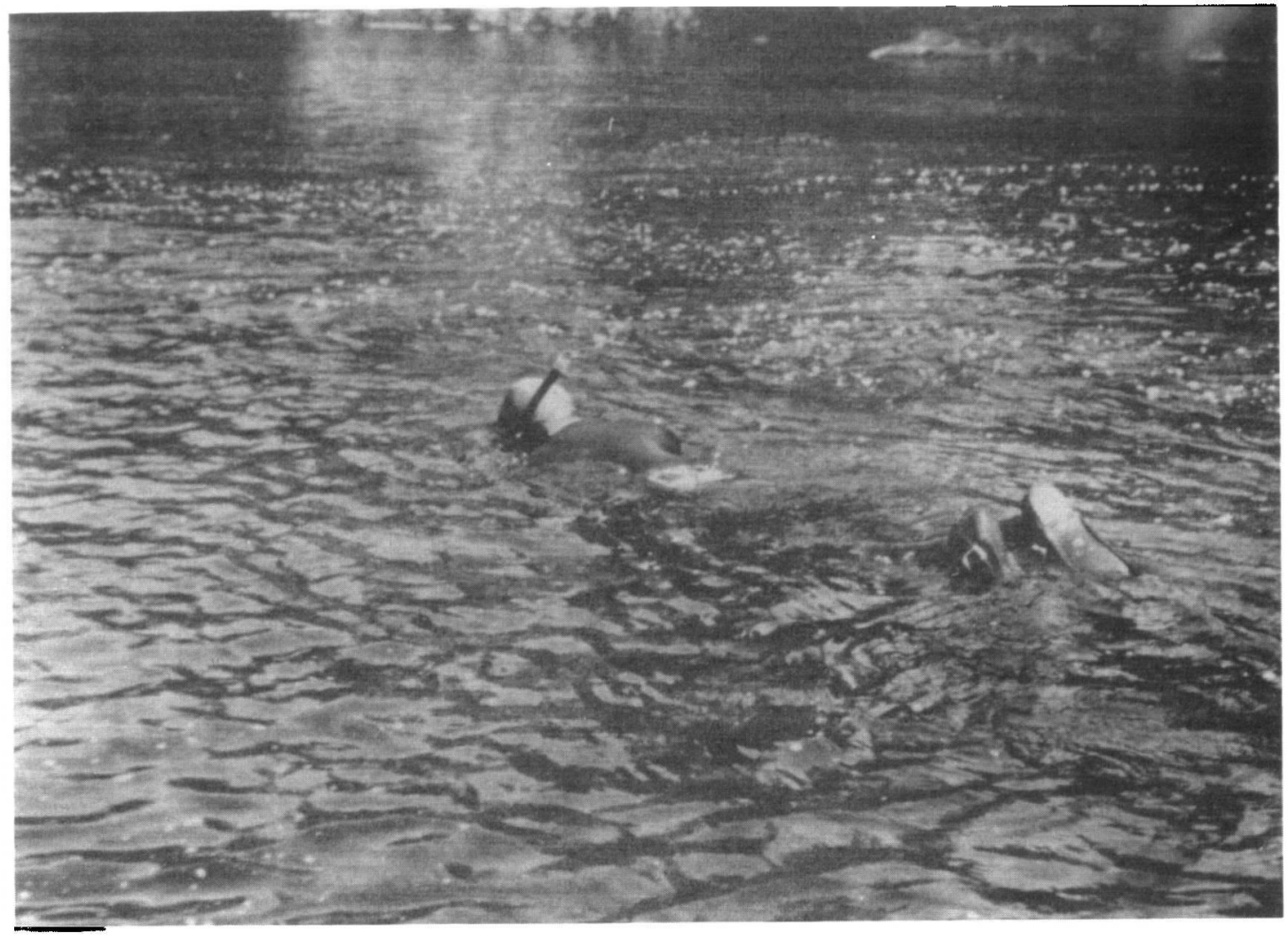

Figure 3 : Plongeur en apnée observant le comportement des alevins de saumons atlantiques. Figure 3 : Diver with snorkel watching the behavior of the Atlantic salmons.

\begin{tabular}{|c|c|c|c|c|c|}
\hline \multirow{2}{*}{ Station } & \multicolumn{6}{|c|}{ Séquence d'observation } \\
\cline { 2 - 6 } & \multicolumn{2}{|c|}{1982} & \multicolumn{2}{|c|}{1983} \\
\cline { 2 - 6 } & $0 \mathrm{~h}$ & $24 \mathrm{~h}$ & $48 \mathrm{~h}$ & $0 \mathrm{~h}$ & $24 \mathrm{~h}$ \\
A & 110 & 25 & 20 & 30 & 25 \\
C & 10 & 25 & 20 & - & - \\
D & 10 & 25 & 20 & 30 & 25 \\
E & 45 & 25 & 20 & 30 & 25 \\
& - & - & - & 30 & 25 \\
\hline
\end{tabular}

Tableau 3 : Durée (min) des séquences d'observation par station.

Table 3 : Duration ( $\mathrm{min}$ ) of observation sequences by station. 


\section{RÉSULTATS}

Au moment de leur libération dans la rivière, les alevins se précipitaient immédiatement au fond du cours d'eau et y restaient immobiles. Le comportement territorial et agressif qui leur est habituel (GIBSON, 1973; KEENLEYSIDE et YAMAMOTO, 1962) était absent. Les 500 individus libérés à chaque station occupaient une surface inférieure a $10 \mathrm{~m}^{2}$. La présence de blocs et de galets dans les stations situées dans le rapide offrait des abris permettant aux alevins d'être protégés du courant relativement élevé $\left(18\right.$ à $\left.61 \mathrm{~cm} . \mathrm{s}^{-1}\right)$. Les alevins n'ayant pas trouvé immédiatement d'abris étaient emportés par le courant. En général, après 10 à 20 minutes, les jeunes saumons devenaient plus actifs et quelques jeunes individus commençaient à s'éloigner du lieu de déversement. Des individus ont été vus essayant de s'alimenter en capturant des particules en suspension amenées par le courant 20 minutes après l'introduction. Une prédation a été effectuée par l'omble de fontaine sur les alevins nouvellement introduits (tableau 4). Des ombles d'environ 9 à $14 \mathrm{~cm}$ ont été vus capturant des jeunes saumons aux stations situées au pied du rapide (stations A, B et C) et à la station D située au centre (fig. 2). L'un d'entre eux dévora cinq alevins durant les 30 premières minutes suivant l'introduction. Des

\begin{tabular}{|c|c|c|c|c|c|c|c|c|c|}
\hline \multirow{3}{*}{ Station } & \multicolumn{9}{|c|}{1982} \\
\hline & \multicolumn{3}{|c|}{$0 \mathrm{~h}$} & \multicolumn{3}{|c|}{$24 \mathrm{~h}$} & \multicolumn{3}{|c|}{$48 h$} \\
\hline & $P$ & A & $S$ & $P$ & $A$ & $S$ & $P$ & A & $S$ \\
\hline$A$ & $1 \mathrm{Ta}$ & 1 & 1 & 0 & 0 & 0 & 0 & 0: & 0 \\
\hline B & 0 & 0 & 0 & 0 & 0 & 0 & 0 & 0 & 0 \\
\hline$C$ & 0 & 0 & 0 & 0 & 0 & 0 & 0 & 0 & 0 \\
\hline D & $20 \mathrm{~m}$ & 5 & 2 & 0 & 0 & 0 & 0 & 0 & 0 \\
\hline \multirow{3}{*}{ Station } & \multicolumn{9}{|c|}{1983} \\
\hline & \multicolumn{3}{|c|}{$0 \mathrm{~h}$} & \multicolumn{3}{|c|}{$24 h$} & & & \\
\hline & $P$ & A & $S$ & $\mathbf{P}$ & A & $S$ & & & \\
\hline$A$ & $\begin{array}{ll}1 & 0 \mathrm{~m} \\
2 & \mathrm{Ta}\end{array}$ & $\begin{array}{l}5 \\
0\end{array}$ & $\begin{array}{l}0 \\
0\end{array}$ & 0 & 0 & 0 & & & \\
\hline $\mathrm{C}$ & $\begin{array}{ll}3 & \mathrm{Om} \\
4 & \mathrm{Ta}\end{array}$ & $\begin{array}{r}19 \\
0\end{array}$ & $\begin{array}{l}5 \\
0\end{array}$ & 0 & 0 & 0 & & & \\
\hline D & $1 \mathrm{Ta}$ & 0 & 0 & $3 \mathrm{Ta}$ & 0 & 0 & & & \\
\hline$E$ & $3 \mathrm{Ta}$ & 0 & 0 & 0 & 0 & 0 & & & \\
\hline
\end{tabular}

P: nombre de prédateurs observés

A: nombre d'attaques observées

S: nombre d'alevins capturés
Ta: Tacon

Om: Omble

Tableau 4 : Nombre de prédateurs, d'attaques observées et d'alevins capturés par station et séquences d'observation.

Table 4 : Number of predators, number of attacks and number of fries caught per station and sequences of observation. 
ombles d'environ $7 \mathrm{~cm}$ tentèrent également à maintes reprises de capturer des alevins. Près de 15 tacons ont été aperçus la journée du déversement. Un seul d'entre eux a été vu capturant et dévorant un alevin. Généralement les tacons se maintenaient au même endroit et repoussaient les intrus entrant dans leur territoire alors que les ombles de fontaine maraudaient et fonçaient sur les alevins afin de les capturer.

Après $24 \mathrm{~h}$, aucun groupe important d'alevins n'a été observé. La densité des alevins variait entre 30 et 50 individus par $100 \mathrm{~m}^{2}$ autour de chaque point de déversement. La majorité des individus observés s'alimentaient et étaient beaucoup plus alertes que lors de l'introduction. Il était plus difficile de repérer les alevins car ceux-ci se cachaient entre les pierres ou sous celles-ci. Malgré la présence de quelques tacons (tableau 4), aucun d'entre eux ne chercha à capturer des alevins et aucun omble de fontaine n'a été aperçu (tableau 4).

Après $48 \mathrm{~h}$, les alevins demeuraient en contact ou près du fond et généralement derrière une roche. Ils étaient très alertes et possédaient un comportement territorial et agressif normal.

Des densités variant entre 24 et 34 alevins par $100 \mathrm{~m}^{2}$ ont été observées dans le rapide faisant partie de la zone d'observation (fig. 2) 1 mois après chacun des déversements (LEGAULT, 1985). En 1982, 3 alevins furent capturés dans la section aval du bassin à la station de recapture F (fig. 2) 6 jours après l'introduction. Finalement, près de 3 mois plus tard, 3 alevins ont été capturés dans le petit rapide, à la station de recapture $\mathbf{G}$ (fig. 2) située en aval du site où les déversements ont été effectués. Aucun alevin n'a été capturé à une station d'échantillonnage située à $0,9 \mathrm{~km}$ en amont du lieu d'introduction (LEGAULT, 1985).

\section{DISCUSSION}

Tout comme ELSON (1940) et MC CRIMMON (1954) nous avons noté une forte prédation effectuée par l'omble de fontaine sur les alevins nouvellement implantés. Celui-ci semble devenir un prédateur potentiel pour les jeunes alevins déversés (LF : $3,5 \mathrm{~cm}$ ) dès qu'il atteint une taille d'environ $7 \mathrm{~cm}$. Etant donné que l'omble de fontaine était beaucoup plus abondant dans les zonesd'eauxcalmes et profondes (LEGAULT, 1985), cela expliquerait que la prédation ait été observée principalement dans ce genre d'habitat. Contrairement à SYMONS et HELAND (1978) qui ont effectué leurs observations en laboratoire, en une seule occasion il nous a été donné de voir un tacon capturer un alevin. Les tacons se contentaient de repousser ceux qui pénétraient dans leur territoire.

Le transport des poissons de la pisciculture aux lieux de déversement ainsi qu'une méthode d'introduction inadéquate seraient responsables en grande partie de la prédation effectuée par l'omble de fontaine sur les alevins nouvellement déversés. Le transport des alevins de saumons semble inhiber leur comportement agressif et territorial. Nous avons observé que lorsque les alevins étaient libérés dans le milieu naturel, ceux-ci restaient immobiles au fond du cours d'eau. IIs ne réagissaient plus aux divers stimuli de l'environnement. Ce n'est qu'après 10 à 20 minutes que les alevins devenaient plus actifs et une période de 24 à 48 h s'est écoulée avant que les alevins ne démontrent un comportement agressif et territorial. RIMMER (1975) observa durant le transport une diminution importante du pH et une augmentation du $\mathrm{CO}_{2}$ gazeux, immobilisant ainsi les poissons. De plus, les mouvements constants et multidirectionnels de l'eau, renversant les poissons, seraient probablement un facteur clé causant la fatigue et la désorientation de ceux-ci (RIMMER, 1975). C'est durant les premières heures suivant les déversements que nous avons observé une forte prédation de la part de l'omble de fontaine. Les alevins étant immobiles, désorientés et ne cherchant pas à se cacher, ceux-ci devenaient très vulnérables et sujets à une prédation importante.

Une période de récupération dans la rivière permettrait aux alevins de se remettre des mauvaises conditions existant durant le transport et de retrouver leur agressivité tout en les familiarisant aux conditions de leur nouveau milieu. Ils seraient plus aptes à affronter les conditions naturelles, ce qui pourrait résulter en un taux de survie plus élevé. CRESSWELL et WILLIAMS (1983) démontrèrent en effet qu'une période d'acclimatation en rivière avant la libération se traduit par un taux de survie plus élevé chez la truite brunè (Salmo trutta).

Nous avons observé que les alevins se précipitent immédiatement au fond du cours d'eau lors de leur introduction dans la rivière. Ce comportement a également été observé en laboratoire par SOSIAK (1978) et par SYMONS et HELAND (1978). Cette réaction des alevins leur permet de se maintenir dans des lieux où le courant est élevé, à condition qu'ils y retrouvent des abris tels que des blocs et des galets. Sans ces abris, les alevins sont emportés jusqu'à ce qu'ils rencontrent un environnement où le courant est plus faible, et où l'omble de fontaine est plus abondant que dans les sections d'eau vive (GIBSON 1966, 1973).

D'après SYMONS (1969) et BULLEID (1973) les tacons introduits ne migrent pas d'une façon progressive mais plutôt d'une façon sporadique. Ils migreraient jusqu'au moment où ils rencontreraient un micro-habitat convenable, passant généralement outre lorsque celui-ci est défendu par un occupant. Même si les alevins ont la capacité de migrer sur de longues distances (ELSON, 1975) afin 
de trouver un micro-habitat adéquat et inoccupé, ceux-ci peuvent s'affaiblir et être plus susceptiblesà la prédation lors de leur migration. Contrairement à ELSON (1975), nous n'avons pas observé de migration importante des alevins hors du lieu de leur implantation. La majorité des alevins est demeurée aux environs du site d'introduction. A cet endroit la densité des tacons était faible (LEGAULT, 1985). De plus, en nous basant sur les recommandations de SYMONS et HELAND (1978) concernant lès densités de peuplement des saumons d'élevage, le nombre d'alevins que nous avons déversés faisait en sorte que le site choisi avait une superficie suffisante d'habitat de bonne qualité pour convenir à leurs besoins. Ceci expliquerait donc que les alevins soient demeurés sur les lieux de déversement et que leur migration fût très limitée. Une dispersion adéquate des alevins dans les sections d'eau vive, possédant des abris tels que des blocs et des galets, tout en tenant compte de leur capacité de peuplement, permettrait ainsi de limiter leur migration et par le fait même de diminuer la prédation effectuée par l'omble de fontaine. Il serait donc important de localiser avant une introduction les sections de rivière possédant les caractéristiques répondant aux exigences des alevins et d'estimer leur capacité d'accueil.

Il semble difficile d'envisager une période de récupération pour les alevins avant leur déversement tout en les dispersant dans les sections de rivière répondant à leurs exigences. Selon nos observations, ce n'est qu'après une période de 24 à 48 h que les alevins reprennent un comportement agressif et territorial. Cependant, cette période ne représente pas nécessairement le temps requis afin de récupérer du transport. Nous avons également observé qu'après une période de 10 à 20 minutes. les alevins devenaient plus actifs et que quelques individus commençaient à s'alimenter. Cette courte période serait peut-être suffisante pour permettre une récupération adéquate aux alevins. Il serait dès lors possible de permettre aux alevins de récupérer sur les lieux même des déversements, en changeant l'eau des sacs de transport et en les laissant reposer 10 à 20 minutes avant d'effecter leur dispersion. Dans ce sens, il serait important de déterminer plus précisément la période nécessaire pour que les alevins puissent récupérer de façon satisfaisante du transport.

A notre connaissance, cette étude est la première à avoir tenté d'observer en apnée le comportement et le devenir immédiat des jeunes saumons atlantiques suite à leur déversement dans une rivière naturelle.

Les observations que nous avons effectuées en apnée nous ont permis d'observer le comportement des saumons juvéniles dans des conditions où le poisson est libre de ses mouvements èt où il doit faire face aux diverses conditions environnementales. Ainsi, les observations menées en apnée dans un milieu naturel reflètent mieux la réalité que si elles étaient effectuées au-dessus de la surface de l'eau (McCRIMMON, 1954) ou lorsque les poissons sont confinés dans une rivière artificielle (SOSIAK, 1978 ; SYMONS et HELAND, 1978).

La visibilité est le principal facteur qui limite l'observation. Cependant, dans les zones d'eaux rapides nous avions une visibilité d'environ $1 \mathrm{~m}$. Etant donné que nous pouvions nous approcher à moins de $30 \mathrm{~cm}$ d'un poisson avant que celui-ci semble être importuné, ce facteur avait peu d'importance.

En conclusion, les mauvaises conditions prévalant généralement durant le transport ainsi qu'une méthode d'introduction inadéquate seraient responsables en grande partie de la prédation effectuée par l'omble de fontaine sur les alevins de saumons atlantiques déversés. Cette prédation pourrait être minimisée : (1) en donnant une période de récupération aux alevins afin qu'ils puissent se remettre du transport avant d'être libérés et (2) en dispersant les alevins dans les sections de rivière répondant à leurs exigences tout en respectant leur capacité d'accueil.

\section{REMERCIEMENTS}

Cette étude a été faite grâce à une bourse d'études du programme pour la formation de chercheurs et d'action concertée (FCAC). Nous remercions la direction régionale du Saguenay/Lac Saint-Jean du Ministère du Loisir, de la Chasse et de la Pêche pour nous avoir fourni les ouananiches d'élevage et pour leur aide technique. Messieurs Marc BOUDREAU et Philippe THIBEAULT nous ont assisté lors des observations effectuées en apnée; nous les en remercions.

\section{REFERENCES}

BENSON N.G., 1953. Seasonal fluctuation in the feeding of brook trout in the Pigeon River, Michigan. Trans. Amer. Fish. Soc. 83 : 76-83.

BULLEID M.J., 1973. The dispersion of the hatchery-reared Atlantic salmon (Salmo salar/ stocked into a fishless stream. Ont. Atl. Sal. Foun. Spec. Pub. Ser. $4: 169-179$.

CRESSWELL R.C., R. WILLIAMS, 1983. Post-stocking movements and recapture of hatchery-reared trout released into flowing waters-effect of prior. acclimation to flow. J. Fish Biol. $23: 256-276$. 
ELSON P.F., 1940. Predators of salmo fingerlings in Goldmine Brook. Fish. Res. Bd. Can., MS Rept. Biol. Stns. No. 210. VI: 1-13.

ELSON P.F., 1975. Atlantic salmon rivers, smolt production and optimal spawning : an overview of natural productions. Int. Atl. Sal. Foun. Spec. Pub. Ser. 6 : 96-119.

GIBSON R.J., 1966. Some factors influencing the distributions of brook trout and young Atlantic salmon. J. Fish. Res. Bd. Can. 23 : 977-980.

GIBSON R.J., 1973. Interactions of juvenile Atlantic salmon (Salmo salar L.) and brook trout (Salvelinis fontinalis Mitchill). Int. Atl. Sal. Foun. Spec. Pub. Ser. 4 : 181-202.

HYNES H.B.N., 1972. The ecology of running waters. University of Toronto Press, Toronto (Ont.) $555 \mathrm{p}$.

KEENLEYSIDE M.H.A., 1962. Skin-diving observations of Atlantic salmon and brook trout in the Miramichi River, New Brunswick. J. Fish. Res. Bd. Can. 19: 625-634.

KEENLEYSIDE M.H.A., F.T. YAMAMOTO, 1962. Territorial behavior of juvenile Atlantic salmon (Salmo salar, L.). Behavior 19 : 139-169.

LEGAULT M., 1985. Détermination des répercussions de l'élevage en pisciculture du saumon atlantique (Salmo salar) sur son comportement et ses performances dans une rivière naturelle. Mémoire de maîtrise, Univ. du Québec à Chicoutimi, Chicoutimi (Québec).

Mc CRIMMON H.R., 1954. Stream studies on planted Atlantic salmon. J. Fish. Res. Bd. Can. 11 : 362-403.

RIMMER D.M., 1975. Rearing Atlantic salmon (Salmo salar L.) in fishless lakes of the Matamek river system, Quebec. Thèse de maîtrise, Univ. Waterloo, Waterloo (Ont.).

SOSIAK A.J., 1978. The comparative behaviour of wild and hatchery-reared juvenile Atlantic salmon (Salmo salar L.). Thèse de maîtrise, Univ. New Brunswick, Fredericton (N.-B.).

SYMONS P.E.K., 1969. Greater dispersal of wild compared with hatchery-reared juvenile Atlantic salmon released in streams. J. Fish. Res. Bd. Can. 26 : 1867-1876.

SYMONS P.E.K., M. HELAND, 1978. Stream habitats and behavioural interactions of underyearling and yearling Atlantic salmon (Salmo salar). J. Fish. Res. Bd. Can. 35 : 175.183. 